\author{
I.I.K. Paul iny-Toth \\ Max-Planck-Institut für Radioastronomie \\ Bonn, FRG
}

\title{
1. THE SURVEYS
}

A number of surveys have been carried out at a frequency of $5 \mathrm{GHz}$ at the National Radio Astronomy Observatory (NRAO) and at the MaxPlanck-Institut für Radioastronomie (MPIfR), with the aim of determining the number-flux density relation for the sources detected and also of obtaining their radio spectra and optical identifications. The surveys fall into two categories: first, the strong source (S) surveys, which are intended in due course to cover the whole northern sky and to be complete above a flux density of about $0.6 \mathrm{Jy}$; second, surveys of limited areas of sky down to lower levels of the flux density.

The regions of sky covered by the strong source surveys are shown in Figure 1. Three of these, denoted by S1, S2 and S3, have already

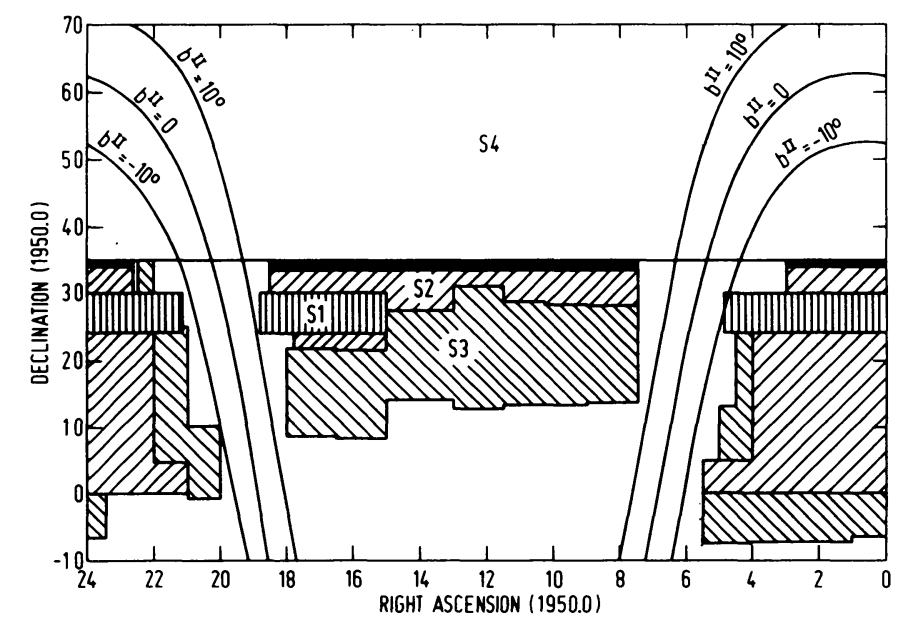

Figure 1 . Areas of sky covered by the $S$ surveys. The narrow strip shaded in black is the area of the "I" survey. 
been reported (Kellermann et al. 1968, Pauliny-Toth et al. 1972, Paul iny-Toth and Kellermann 1972). All three were made with the NRAO 140-ft. telescope and consisted of a fast finding survey, followed by reobservation of the sources detected. In the case of the more recent S4 survey (Pauliny-Toth et al. 1977), the finding survey was made with the NRAO 300-ft. telescope and the sources were reobserved with the MPIfR 100-m antenna. This survey covers the sky between declinations of $35^{\circ}$ and $70^{\circ}$ and is somewhat more sensitive than the other $S$ surveys.

Further data of comparable sensitivity for the southern sky are provided by the Parkes $2.7 \mathrm{GHz}$ surveys, a number of which are complete above a flux density of $0.35 \mathrm{Jy}$ at $2.7 \mathrm{GHz}$ (Wall et al. 1971, Shimmins 1971, Shimmins and Bolton 1972b and 1974, Bolton and Shimmins 1973, Bolton et al. 1975, Wall et al. 1976). Flux densities at $5 \mathrm{GHz}$ for the sources in these surveys have been reported either in the above references or by Shimmins and Bolton (1972a) and Shimmins et al. (1969). The distribution of the spectral indices of these sources, derived from the data at 2.7 and $5 \mathrm{GHz}$ shows that a sample of sources having a flux density $\mathrm{S}(2.7 \mathrm{GHz})>0.35 \mathrm{Jy}$ would be virtually complete at $5 \mathrm{GHz}$ above $0.6 \mathrm{Jy}$. This sample, taken from the Parkes survey, covers a solid angle of $2.6 \mathrm{sr}$ when regions of overlap with the $S$ surveys, areas within the Magellanic clouds, or areas within $10^{\circ}$ of the galactic plane are excluded.

Table I gives the solid angle of sky covered by each strong source survey, as well as the number of sources found above the given limit of completeness, both for the whole survey, and for the areas in the south and north galactic hemispheres having galactic latitudes greater than $10^{\circ}$. In the Table, the S3 survey, which overlapped part of the more sensitive S4 survey, has been correspondingly reduced in area, while sources from the "I" survey (see below) having flux densities greater than $0.5 \mathrm{Jy}$ have been used to fill the gap between the S4 and the other $\mathrm{S}$ surveys.

Table I

$5 \mathrm{GHz}$ strong source surveys.

\begin{tabular}{|c|c|c|c|c|}
\hline Survey & $\begin{array}{l}\text { Solid a } \\
\text { Total }\end{array}$ & $\begin{array}{l}\text { e covered } \\
\mathrm{bII}<-10^{0}\end{array}$ & $\begin{array}{l}\text { Number of sources } \\
\mathrm{b}^{\mathrm{II}}{ }_{>+10^{0}}\end{array}$ & $\begin{array}{l}\text { Completeness } \\
\text { Limit (Jy) }\end{array}$ \\
\hline $\begin{array}{l}\text { S1 } \\
\text { S2 } \\
\text { S3 } \\
\text { S4 } \\
\text { I } \\
\text { PKS }\end{array}$ & $\begin{array}{l}0.273 / 23 \\
0.973 / 136 \\
1.059 / 123 \\
1.713 / 208 \\
0.074 \quad \\
2.604 / 302\end{array}$ & $\begin{array}{lr}0.185 / & 14 \\
0.760 / 100 \\
0.399 / & 51 \\
0.296 & 42 \\
0.015 & 42 \\
1.922 / 217\end{array}$ & $\begin{array}{l}0.088 / \\
0.213 / .36 \\
0.660 / 72 \\
1.416 / 66 \\
0.059 / 65 \\
0.682 / \quad 85\end{array}$ & $\begin{array}{l}0.8 \\
0.6 \\
0.6 \\
0.5^{+} \\
0.6\end{array}$ \\
\hline
\end{tabular}

+ Numbers of sources are given for $S>0.6 \mathrm{Jy}$. The total number above $0.5 \mathrm{Jy}$ is 269 .

The complete sample of sources stronger than 0.6 Jy thus contains 769 sources and covers $6.48 \mathrm{sr}$, that is, more than half of the sky at galactic latitude greater than $10^{\circ}$. 
The surveys of limited areas of sky, complete down to lower flux densities, consist of:

a) an "intermediate source" (I) survey, complete down to $0.25 \mathrm{Jy}$ and covering $0.079 \mathrm{sr}$ (Pauliny-Toth et al. 1972),

b) a deep (D) survey, made with the 300-ft. telescope, and covering $3.77 \times 10^{-2} \mathrm{sr}$ down to a limiting flux density of 0.067 Jy (Davis 1971), and

c) two deeper surveys made with the MPIfR 100-m telescope. The first of these extends over an area of $4.53 \times 10^{-3} \mathrm{sr}$ near the North Celestial Pole (Pauliny-Toth, Witzel and Baldwin 1977) and is believed to be complete to $0.020 \mathrm{Jy}$; the second covers ten small regions near declination $30^{\circ}$ and is complete to $0.015 \mathrm{Jy}$ over an area of $2.91 \times 10^{-3} \mathrm{sr}$ (Pauliny-Toth, Witzel and Preuß 1977). These surveys contain 59 and 58 sources above their respective limiting flux densities.

For all these surveys, measurements of the flux densities of the sources at other frequencies have been, or are being made. Virtually all the sources in the $S$ surveys have been observed at $2.7 \mathrm{GHz}$, either with the 300-ft. telescope (Davis, unpublished data) or with the 100-m telescope (Pauliny-Toth et a1. 1977), and measurements of most of the sources at $10.7 \mathrm{GHz}$ are available (Pauliny-Toth et al. 1972, and 1977). Sources from the $D$ survey have been measured at both these frequencies (Pauliny-Toth et a1. 1974), and observations of sources from the MPIfR deep surveys are in progress (Davis 1977).

Optical identifications for sources in the first three $S$ surveys have been reported with the survey results, and also by Johnson (197.4) for the S3 survey. For sources in the S4 survey, accurate optical positions for objects in the fields of those sources which had not previously been identified have been measured (Kühr 1977) and identifications have been suggested. These new identifications are based on accurate radio positions measured by Fomalont with the NRAO threeelement interferometer for about one-third of the sources, and on positions measured with the 100-m telescope with an accuracy of about 3.5 arc sec for a further third, the rest having been previously identified. Suggested optical identifications are thus available for almost all sources in the complete $S$ survey sample.

\section{THE SOURCE COUNTS}

The composite source counts at $5 \mathrm{GHz}$ are shown in Figure 2 in the form of a differential plot, normal ised to a uniform Euclidean model having an integral count $N_{0}=60 \mathrm{~s}^{-1.5}$. The flux density intervals within each survey have been chosen so that this model should give approximately the same number of sources in each interval.

For the stronger sources, having $S(5 \mathrm{GHz})>0.6 \mathrm{Jy}$, a numberflux relation of the form $N=K S X$ has been fitted to the ungrouped data, using the method of maximum likelihood (Crawford et al. 1970), 


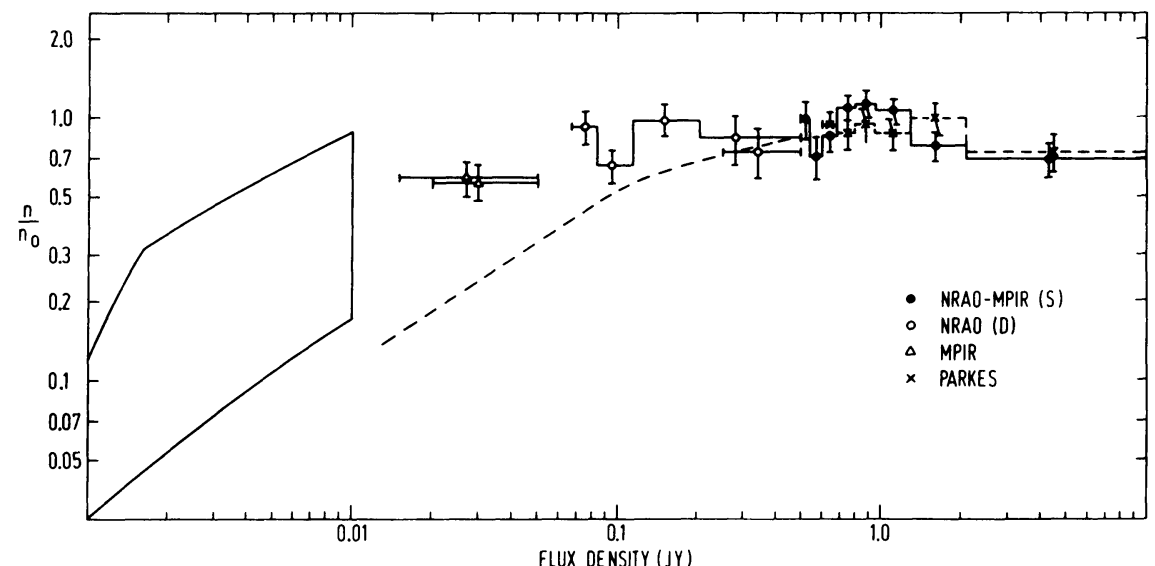

Figure 2. Differential counts at $5 \mathrm{GHz}$, normalised to $\mathrm{N}_{0}=60 \mathrm{~s}^{-1.5}$.

with the results shown in Table II.

Table II

Number-flux relation for $\mathrm{S}(5 \mathrm{GHz})>0.6 \mathrm{Jy}$

Sample $k \quad x \quad$ Number of sources

\begin{tabular}{llll}
\hline A11 data & $50.9 \pm 1.6$ & $-1.66 \pm 0.06$ & 769 \\
S surveys & $51.0 \pm 2.4$ & $-1.71 \pm 0.08$ & 467 \\
Parkes & $52.0 \pm 2.5$ & $-1.57 \pm 0.09$ & 302
\end{tabular}

The difference between the slope of the counts for the stronger sources and the Euclidean value is formally significant at a level of about $2.5 \sigma$. It must be noted, however, that this difference is about the same as that between the slopes for the $S$ surveys and the Parkes sample, and that an even larger difference exists between the slopes of the source counts in the two galactic hemispheres (Section 5). The real significance of the steep slope found for the stronger sources at $5 \mathrm{GHz}$ is thus far from clear. If it is due to evolution of the sources, (e.g. Longair 1974), then the evolution differs for different directions in the sky. If, on the other hand, it is caused by a local "hole" in their distribution (e.g. Pauliny-Toth and Kellermann 1974) then the cosmological interpretation for the redshifts of a large fraction of the sources must be abandoned.

In the intermediate range of flux densities $(0.1$ to $1.5 \mathrm{Jy})$, the slope of the number counts is close to -1.5 . Below this range, the deep survey data indicate that it flattens to about -1.3. The further convergence of the counts is supported by the $P(D)$ analys is of Wall and Cooke (1975) whose limits on the number-flux relation between 0.01 and 0.001 Jy are indicated by the area outlined in Figure 2 . 
The observed convergence of the source counts at $5 \mathrm{GHz}$ is, however, not consistent with the prediction of the evolutionary model of Fanaroff and Longair (1973), shown as a dashed curve in Figure 2. The model, which is based on the luminosity function and sepctral index distribution at $178 \mathrm{MHz}$, predicts counts at $5 \mathrm{GHz}$ for the weak sources which are consistently lower than those observed, the discrepancy reaching a factor of about 3 at the limit of the deep surveys. This discrepancy has also been noted by Davis and Taubes (1974), on the basis of their $P(D)$ analysis of data from the $D$ survey. The model of Fanaroff and Longair predicts that the fraction of sources having flat or inverted spectra between $5 \mathrm{GHz}$ and $178 \mathrm{MHz}$ should decrease with $\mathrm{S}(5 \mathrm{GHz})$, except for the strongest sources. Such a decrease is in fact observed (Pauliny-Toth and Kellermann 1972, Condon and Jauncey $1974)$, down to $\mathrm{S}(5 \mathrm{GHz})$ in the range 0.25 to $0.067 \mathrm{Jy}$. As Davis and Taubes have suggested, a possible explanation for the discrepancy in the source counts is that the predicted decrease in the flat-spectrum population does not continue with a further decrease in the flux density. A direct test of this suggestion will be provided by the measurements of the deep survey sources at other frequencies.

\section{IDENTIFICATION CONTENT}

The statistics of the identifications for the complete $S$ survey sample, excluding a few optically crowded fields and two sources identified with planetary nebulae, are given in Table III for sources identified with quasistellar objects (QSS), radio galaxies (GAL) or lying in empty fields (EF).

Table III

Identification content of the $S$ surveys

\begin{tabular}{|c|c|c|c|c|c|c|}
\hline \multirow{2}{*}{$\begin{array}{l}\text { Flux density } \\
\text { range (Jy }\end{array}$} & \multicolumn{2}{|c|}{ GAL } & \multicolumn{2}{|c|}{ QSS } & \multicolumn{2}{|c|}{$E F$} \\
\hline & $N$ & $\%$ & $N$ & $\%$ & $N$ & $\%$ \\
\hline $\begin{array}{r}0.6 \text { to } 0.8 \\
0.8 \text { to } 1.3^{+} \\
>1.3^{+}\end{array}$ & $\begin{array}{l}57 \\
62 \\
50\end{array}$ & $\begin{array}{l}34 \\
35 \\
44\end{array}$ & $\begin{array}{l}60 \\
77 \\
48\end{array}$ & $\begin{array}{l}36 \\
44 \\
43\end{array}$ & $\begin{array}{l}51 \\
38 \\
15\end{array}$ & $\begin{array}{l}30 \\
21 \\
13\end{array}$ \\
\hline
\end{tabular}

${ }^{+}$Includes sources from SI survey.

The identification content of the strong source sample is very different from that found for metre-wavelength surveys: a much larger fraction of the sources are identified with QSS. This difference is related to the spectral-index distribution (Section 4) which shows that a larger fraction of the sources in high-frequency surveys have flat or inverted spectra and that most of these sources are QSS.

The number-flux relation for the sources identified with quasistellar objects or radio galaxies has, in each case, a slope close to -1.5 . Whereas that of sources lying in empty fields has the much steeper slope of about -2.2. The significance of this result depends, 
of course, on the nature of the objects associated with empty fields: whether these are a class of subluminous objects, or whether they are galaxies or quasistellar objects beyond the limit of the Palomar Sky Survey. The spectral index data (Section 4) indicate that the empty field sources may be a mixture of the two latter classes in roughly equal numbers, in which case the number-flux relation for both QSS and radio galaxies has a slope similar to the overall slope found for the $S$ surveys. This would be surprising, in view of the different volumes of space over which the two classes of objects are presumably distributed, and deep optical searches for objects in the empty fields are desirable.

\section{THE SPECTRAL INDEX DISTRIBUTION}

The distribution of the two-point spectral index, $\alpha$, defined by $\alpha=\log \left|S\left(\nu_{2}\right) / S\left(\nu_{1}\right)\right| / \log \left(\nu_{2} / \nu_{2}\right)$ and obtained from the flux densities measured at 2.7 and $5 \mathrm{GHz}$, is shown in Figure 3 for the $\mathrm{S}$ survey sample.

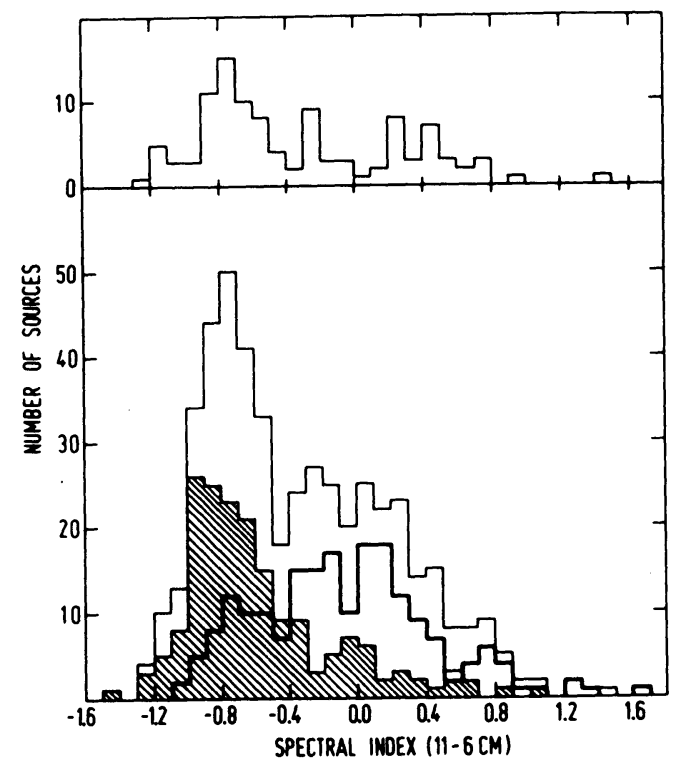

Figure 3. Spectral index distribution for the $S$ survey sources. Top, EF, bottom, all sources, QSS (heavy line), GAL (shaded).

The separate distributions for radio galaxies, quasistellar sources and empty fields are also shown. The distribution is double-peaked, the narrow peak near $\alpha=-0.8$ being due largely to the radio galaxies and the broader peak near $\alpha=0$ to the QSS. The median index, $\bar{\alpha}$, and the fraction of sources which have $\alpha>-0.5$ are given in Table IV for the whole sample, for the different identification classes and for several ranges of the flux density. 
Table IV

Spectral index distribution for the $S$ surveys

\begin{tabular}{lccc}
\hline C1ass & \multicolumn{1}{c}{$\bar{\alpha}^{+}$} & $f$ & Number \\
\hline A11 sources & $-0.45 \pm 0.05$ & $0.53 \pm 0.03$ & 467 \\
QSS & $-0.11 \pm 0.04$ & $0.75 \pm 0.06$ & 187 \\
GAL & $-0.71 \pm 0.03$ & $0.29 \pm 0.04$ & 169 \\
EF & $-0.49 \pm 0.06$ & $0.50 \pm 0.07$ & 104 \\
$0.68>S>0.60$ & $-0.56 \pm 0.14$ & $0.48 \pm 0.08$ & 73 \\
$0.80>S>0.68$ & $-0.46 \pm 0.06$ & $0.52 \pm 0.07$ & 99 \\
$0.96>S>0.80$ & $-0.55 \pm 0.10$ & $0.47 \pm 0.07$ & 95 \\
$1.30>S>0.96$ & $-0.29 \pm 0.13$ & $0.57 \pm 0.07$ & 102 \\
$2.10>S>1.30$ & $-0.31 \pm 0.08$ & $0.59 \pm 0.09$ & 68 \\
S $>2.10$ & $-0.50 \pm 0.08$ & $0.50 \pm 0.09$ & 56 \\
\hline
\end{tabular}

the error in $\bar{\alpha}$ is the mean of the ranges on each side of the median which contain $\sqrt{N} / 2$ sources.

The QSS and radio galaxies have very different distributions: 75 percent of the former, but only 29 percent of the latter have the flat or inverted spectra characteristic of compact, opaque radio sources. The empty fields show an intermediate distribution which can be closely reproduced by a mixture of radio galaxies and quasistellar sources in a ratio of $5: 4$. The situation is different from that found for $10 w-$ frequency surveys, in which the empty field sources have spectra similar to those of radio galaxies (Bolton 1966, Pauliny-Toth and Kellermann 1968).

There is no obvious dependence of $\bar{\alpha}$ on the flux density, even when the spectral index data (Pauliny-Toth et al. 1974) for the D survey sources are included, and consistent with this, there is no difference in the slope of the number counts for sources with steep or flat spectra. By contrast, the spectral index between $5 \mathrm{GHz}$ and 408 or 318 MHz does show a dependence on flux density (Pauliny-Toth and Kellermann 1972, Condon and Jauncey 1974), in the sense that weaker sources tend to have steeper spectra over this range of frequency. Such a dependence is predicted by the model of Fanaroff and Longair (1973), or, more simply, from the number-flux relation and spectral index distribution at longer wavelengths (Paul iny-Toth and Kel lermann 1972), but it probably does not continue below $\mathrm{S}(5 \mathrm{GHz}) \sim 0.1$ Jy (Section 2).

\section{ANISOTROPY}

The difference in the slope of the number-flux relation for the stronger sources in the $S$ surveys on the one hand, and the Parkes surveys on the other, has been pointed out in Section 2. This difference is even larger when the counts in the northern and southern galactic hemispheres are considered, as Table $V$ shows. 
Table V

Number flux relation for sources having $\mathrm{S}(5 \mathrm{GHz})>0.6 \mathrm{Jy}$ in the two galactic hemispheres

\begin{tabular}{lcccccc}
\hline & \multicolumn{3}{c}{$b^{I I}<-10^{0}$} & \multicolumn{3}{c}{$b^{I I}>+10^{0}$} \\
Sample & $K$ & $x$ & $N / s r$ & $K$ & $x$ & $N / s r$ \\
\hline A1 1 data & $55.1 \pm 2.2$ & $-1.54 \pm .08$ & $121 \pm 6$ & $47.2 \pm 2.3$ & $-1.80 \pm .10$ & $119 \pm 6$ \\
S surveys & $60.7 \pm 3.5$ & $-1.51 \pm .11$ & $131 \pm 10$ & $46.0 \pm 2.9$ & $-1.83 \pm .12$ & $117 \pm 7$ \\
Parkes & $50.9 \pm 2.9$ & $-1.56 \pm .11$ & $113 \pm 8$ & $56.2 \pm 5.0$ & $-1.56 \pm .17$ & $125 \pm 13$ \\
\hline
\end{tabular}

The difference between the values of the slope $x$ in the two hemispheres for the whole sample is significant at the $2 \sigma$ level, and is due to the $S$ surveys alone: the Parkes surveys give a slope which is close to the Euclidean value in both hemispheres. When the $S$ surveys are considered separately, the anisotropy is present in each of them, albeit with reduced significance, so that one may conclude, first, that the effect is real, and second, that the angular scale of the anisotropy is large.

The anisotropy appears to be present to some extent for all the identification classes, but is most marked for the radio galaxies: of the sources having $S(5 \mathrm{GHz})>1.3 \mathrm{Jy}$, the number identified with radio galaxies is $20.5 \pm 3.5 \mathrm{sr}^{-1}$ and $8.6 \pm 1.9 \mathrm{sr}^{-1}$ in the south and north galactic hemispheres, respectively. The observed anisotropy in the source counts is therefore at least partly due to a relative deficiency of bright radio galaxies in the north galactic hemisphere (within the $S$ survey regions). There is no significant difference in the spectral index distribution between the two hemispheres, for the sample as a whole. There is some indication that the spectral index distributions for the $S$ surveys differ: the median indices are $-0.55 \pm 0.05$, $-0.30 \pm 0.10$ and $-0.40 \pm 0.08$ for the S2, S3 and S4 surveys respectively, and the S2 survey does not show the double-peaked distribution of $\alpha$ seen for the other two surveys. Furthermore, the low-frequency spectral index, $\alpha(5 \mathrm{GHz}-408 \mathrm{MHz})$, shows anisotropy, in that the slope of the number-flux relation for sources having $\alpha(5 \mathrm{GHz}-408 \mathrm{MHz})>-0.5$ is steeper in the north galactic hemisphere than in the south. This last effect may be connected with the previously mentioned deficiency of bright galaxies in the north.

For the weakest sources in the survey, there is no evidence at present for any anisotropy: the number counts from the two deep MPIfR surveys agree almost exactly, and the numbers of sources in the separate regions covered by the second of these surveys are consistent with a random distribution.

\section{SUMMARY}

The $5 \mathrm{GHz}$ source counts show the following features:

(i) a slope of -1.5 over a wide range of source density $\left(20 \mathrm{sr}^{-1}\right.$ to 
$\left.2 \times 10^{3} \mathrm{sr}^{-1}\right)$,

(ii) a steeper slope, of -1.66 for the stronger sources,

( $i$ ii) a flattening of the slope to about -1.3 for source densities greater than $2 \times 10^{3} \mathrm{sr}^{-1}$,

(iv) an excess of faint sources over the numbers given by the model of Fanaroff and Longair (1973),

(v) a marked anisotropy in the counts for the stronger sources, the slope being significantly steeper than -1.5 only in the area having $\mathrm{bII}>100$ and Declination $>10^{\circ}$, and being partly due to a deficiency of bright galaxies in this region,

(vi) anisotropy in the spectral index distribution of the sources.

The anisotropy observed for the strong sources, the persistence of a slope close to the Euclidean value, and the source counts for the faint sources suggest that the present evolutionary models are not adequate to explain the high-frequency source counts.

\section{ACKNOWLEDGEMENT}

I wish to thank Drs. M. Davis, E. Fomalont, K. Kellermann, E. Preuss and A. Witzel, as well as Mr. H. Kühr, who have all contributed to the measurements on which this paper is based.

\section{REFERENCES}

Bolton, J.G. 1966, Nature 211, 917.

Bolton, J.G. and Shimmins, A.J. 1973, Australian J. Phys. Astrophys. Suppl. 30, 1 .

Bolton, J.G., Shimmins, A.J., Wall, J.V. and Butler, P.W. 1975, ibid. 34,33 .

Condon, J.J. and Jauncey, D.L. 1974, Astron. J. 79, 1220.

Crawford, D.E., Jauncey, D.L. and Murdoch, H.S. T970, Astrophys. J. $162,405$.

Davis, M.M. 1971, Astron. J. 76, 980.

Davis, M.M. and Taubes, C. 1974, Bull. American Astron. Soc. 6, 485.

Fanaroff, B.L. and Longair, M.S. 1973, Monthly Not. Roy. Astrōn. Soc. $161,393$.

Johnson, K.H. 1974, Astron. J. 79, 1006.

Kellermann, K.I., Pauliny-Toth, I.I.K. and Davis, M.M. 1968, Astrophys. Letters 2, 105.

Kühr, H. 1977, in preparation.

Longair, M.S. 1974, IAU Symposium No. 63, "Confrontation of Cosmological Theories with Observational Data", p. 93, pub1. D. Reidel, Holland, ed. M.S. Longair.

Pauliny-Toth, I.I.K. and Kellermann, K.I. 1968, Astron.J. 73, 953.

Pauliny-Toth, I.I.K. and Kellermann, K.I. 1972, ibid. $77, \overline{797}$.

Pauliny-Toth, I.I.K. and Kellermann, K.I. 1974, IAU Symposium No. 63, "Confrontation of Cosmological Theories with Observational Data", p.111, pub1. D. Reidel, Holland, ed. M.S. Longair. 
Paul iny-Toth, I.I.K., Kel lermann, K.I., Davis, M.M., Fomalont, E.B., and Shaffer, D.B. 1972, Astron.J. 77, 265.

Pauliny-Toth, I.I.K., Preuss, E., WitzeT, A., Kühr, H., Davis, M.M., Fomalont, E.B. and Kellermann, K.I. 1977, in preparation.

Pauliny-Toth, I.I.K., Witzel, A. and Baldwin, J.E. 1977, in preparation.

Pauliny-Toth, I.I.K., Witzel, A. and Preuss, E. 1974, Astron. Astrophys. 35,421 .

Pauliny-Toth, I.I.K., Witzel, A. and Preuss, E. 1977, in preparation.

Shimmins, A.J. 1971, Australian J. Phys. Astrophys. Suppl. $21,1$.

Shimmins, A.J. and Bolton, J.G. 1972a, ibid. 23, 1.

Shimmins, A.J. and Bolton, J.G. 1972b, ibid. $26,1$.

Shimmins, A.J. and Bolton, J.G. 1974, ibid. 32, 1.

Shimmins, A.J., Manchester, R.N. and Harris, B.J. 1969, ibid. 8, 1. Wal1, J.V. and Cooke, D.J. 1975, Monthly Not. Roy. Astron. Soc. 171,9. Wal1, J.V., Shimmins, A.J. and Merkelijn, J.K. 1971, Australian J. Phys. Astrophys. Supp1. 19,1.

Wall, J.V., Bolton, J.G., Wright, A.E., Savage, A. and Vander Hagen, J. 1976, Australian J. Phys. Astrophys. Supp1. 39, 1.

\section{DISCUSSION}

P. Véron: You have said that in one of the instalments of the $5 \mathrm{GHz}$ survey, there is a deficiency of flat spectrum sources. Could you not say just as well that there is an excess of steep spectrum sources?

Witzel: We feel that there is rather a deficiency of flat spectra sources in S2, since in contrast to the double peaked distribution in S3 and S4 there is only one peak (at the steep spectra side) with a tail on the flat spectra side visible in $\mathrm{S} 2$.

Bolton: The difference between the $6 \mathrm{~cm}$ data and my own probably arises from the fact that most sources with their flux maxima at $\mathrm{cm}$ wavelengths have their maxima at wavelengths near $6 \mathrm{~cm}$. Thus a positive spectral index source between 11 and $6 \mathrm{~cm}$ becomes a negative spectral index source between $6 \mathrm{~cm}$ and $3 \mathrm{~cm}$. Thus the clear discrimination between QSO's and Galaxies at $11 \mathrm{~cm}$ becomes somewhat obscured at $6 \mathrm{~cm}$ if the spectra are based on the shorter wavelength data only.

THE EFFECT OF SOURCE VARIABILITY ON SAMPLE STATISTICS

$$
\text { G.D. Nicolson }
$$

At centimetre wavelengths a large fraction of radio sources have flat radio spectra. Because these sources also exhibit large flux density variations the statistical properties of complete samples of radio sources will fluctuate with time. This effect has been investigated by constructing samples which are instantaneously complete above $2.4 \mathrm{Jy}$ at $2.3 \mathrm{GHz}$ by combining surveys at several epochs with variability data measured for individual sources. 
The results show that between 1969.5 and 1975.5 sources with $\mathrm{b} \leqslant-10^{\circ}$ had a constant source count slope of $1.95 \pm 0.26$ while the slope for sources with $b \geqslant 10^{\circ}$ varied between $1.49 \pm 0.23$ and $1.68 \pm$ 0.24. This was caused by fluctuations in the number of sources with flat radio spectra at different times and in particular for the region $b \geqslant 10^{\circ}, \alpha \geqslant 13^{\text {h }}$ the fraction changed from $29 \% \pm 9 \%$ to $9 \% \pm 6 \%$ over five years compared with a time averaged fraction of $26 \% \pm 4 \%$ for the entire sample. The statistical significance of the apparent anisotropies therefore changes with time and the reality of these anisotropies should be viewed with caution.

Mills: I believe that the question of variability is quite irrelevant. It merely gives different samples with the same statistics. Averaging samples taken at different times cannot help to beat the uncertainty.

Wall: Nelson Schuch (MRAO, Cambridge) has done a numerical experiment to examine the effects of intensity variations on the form of the high flux density and of Source counts at high frequencies. He has made the extreme assumption that the variability index distribution derived at Algonquin Radio Observatory is applicable to all sources in high frequency surveys, and in a random number experiment has derived "source counts" at 200 different epochs, starting from a) invented samples with integral slopes of -1.5 and -1.8 , and b) the NRAO $5 \mathrm{GHz}$ survey list. In all cases the different epoch slopes lie within the $\pm 1 \sigma$ statistical errors obtained from maximum fitting to the original sample. Is this consistent with your observations?

Nicolson: Yes.

\title{
COSMOLOGICAL IMPLICATIONS OF VARIABILITY
}

\author{
Jeffrey D. Scargle
}

Two cosmological effects have been investigated using the systematic survey of radio source variability contained in the Algonquin Radio observatory data. In any expanding cosmology, or any cosmology in which the redshift is Doppler or gravitational in origin, the time-scale for variability should vary as $1+z$. Although there is much scatter in the data, the effect has probably been detected, and the "tired light cosmology" can be ruled out at the 968 confidence level - unless evolutionary effects or observational selection have produced the observed relation

$$
T=T_{0}(1+z) 0.82 \pm 0.38
$$

The second cosmological effect is that some quantity derived from the luminosity variations of QSO's may possibly serve as a more reliable standard candle than does the luminosity at a randomly selected time, which has previously been used for radio-magnitude/redshift tests. Various such quantities do show reduced scatter in the magnitude/ 
redshift plane, but possible selection effects, and as shown by Petrosian, the luminosity function and its possible evolution, prevent useful cosmological information from being derived from any such relation. The detection of the wavelength dependence of the arrival time of variations, for most radio sources studied, lends some weight to the expanding plasma cloud model for the variations. 